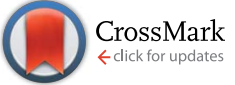

Cite this: RSC Adv., 2017, 7, 15408

Received 14th November 2016 Accepted 25th February 2017

DOI: 10.1039/c6ra26759a

rsc.li/rsc-advances

\section{A solid polymer microneedle patch pretreatment enhances the permeation of drug molecules into the skin}

\begin{abstract}
Qiu Yu Li, Jia Nan Zhang, Bo Zhi Chen, Qi Lei Wang and Xin Dong Guo*
Solid microneedles (MNs) for skin pretreatment have attracted considerable attention in recent years due to their ability to increase skin permeability. There are several approved cosmetic products using microneedles in the market including Dermaroller, silicon MNs, etc. However, a safer MN which is made of biodegradable polymers hasn't been commercialized. Solid polymer MNs still need the support of theoretical data to realize the prospect of mass production and clinical application. In this work, we aim to employ polylactic acid (PLA) MNs to systemically investigate the mechanical and stability properties of the microneedles and the effect of $\mathrm{MN}$ dimensions, drug concentration, the viscosity of drug formulation and the administration time of drug on the skin on the drug permeation into the pretreated skin. Multiple applications test demonstrated MNs with the height of $600 \mu \mathrm{m}$ possessed good mechanical stability performance. $800 \mu \mathrm{m}$ depth microneedle and microneedle with the density of $256 \mathrm{MNs}$ per $\mathrm{cm}^{2}$ were most conductive to enhance the drug permeation. In addition, the increasing of drug concentration could increase the permeation amount of drug, but not affected on drug permeation rate. With the increase of drug viscosity, the drug permeation amount was decreased. To prolong the administration time of drug on the skin at $1 \mathrm{~h}$, the drug permeation amount achieved a stable value and essentially unchanged after $1 \mathrm{~h}$. Finally, the permeation effect induced by the MNs was demonstrated by insulin delivery in vivo. The blood glucose levels of diabetic mice were reduced to $29 \%$ of initial level at $5 \mathrm{~h}$ due to the increased permeability of insulin to the skin after MNs insertion. In conclusion, the biodegradable polymer solid microneedles can painlessly pierce the stratum corneum and accelerate the absorption of drug and active ingredients.
\end{abstract}

\section{Introduction}

Transdermal drug delivery systems are widely used to delivery drugs into the body across the skin. ${ }^{1,2}$ This method offers many important advantages as compared to the oral route, for example it can avoid the hepatic first-pass effect caused by the mouth to improve bioavailability, and reduce the risk of needlestick injuries and pain associated with injections to increase patient compliance. The stratum corneum of the skin acts as a barrier that prevents most drugs or therapeutic agents to penetrate into the body. ${ }^{3}$ Only small lipophilic molecule drugs ( $<400 \mathrm{Da}$ ) generally can cross the skin at therapeutic rates, but the dose of these molecule drugs penetrating through the skin is relatively low. ${ }^{4}$ Conventional approaches have been reported to alter the barrier of the stratum corneum to enhance some small molecule drugs and other biological therapeutic drugs especially macromolecular protein drugs transport across the skin. Some of these approaches include chemical enhancers, ${ }^{5}$

Beijing Laboratory of Biomedical Materials, College of Materials Science and Engineering, Beijing University of Chemical Technology, Beijing, 100029, $P . R$. China.E-mail: xdguo@buct.edu.cn thermal methods, ${ }^{6}$ electroporation, ${ }^{7}$ iontophoresis, ${ }^{8}$ sonophoresis, ${ }^{9,10}$ and laser ablation. ${ }^{11}$ However, chemical enhancers do not seem to be broadly beneficial to strengthen biopharmaceuticals and vaccines across the skin because they can cause skin irritation and other security issues. Meanwhile physical methods mentioned above typically depend on using sophisticated devices which are comparatively costly, large and require special training for patients to use. And more work is still needed to investigate their latent impact on clinical practice to date. ${ }^{7,12}$ Therefore, a new concept, a microneedle (MN) array with micro-sized needles, was introduced to merge the advantages of transdermal delivery and parenteral delivery. ${ }^{13}$ After applying and removing the MNs, the stratum corneum could be penetrated, forming microchannels with no pain and no bleeding that facilitate the drug permeation into the skin. Due to the superiorities of the $\mathrm{MN}$, it has recently emerged as a novel transdermal drug delivery system and received much attention.

MNs have been made primarily by using the technologies of the microelectronics industry to produce arrays on a silicon wafer ${ }^{14,15}$ metal ${ }^{16-18}$ and polymer MNs. ${ }^{19-21}$ Solid MNs have been shown to increase skin permeability by up to four orders of magnitude for compounds ranging in size from small 
molecules, proteins to nanoparticles. ${ }^{22-24}$ Most MNs previously presented are made of silicon or metal, both of which have received limited promotion in the field of transdermal drug delivery due to the nature limitations of the materials themselves. Silicon MNs are relatively expensive, fragile, and unproven as a biocompatible material, and the metal MNs could cause skin problems. ${ }^{25,26}$ Polymer MNs are usually made of biodegradable polymers using a mold-based fabrication method. The biocompatible materials and the fabrication method lend the polymer MNs themselves to be safe, inexpensive, and easy for mass production. In recent years, various biodegradable polymers have been used in the development of solid MNs and applied to the pretreatment of the skin, such as polystyrene, ${ }^{27}$ polyvinyl pyrrolidone, ${ }^{28}$ polycarbonate, ${ }^{29}$ photoresist (SU-8) resin ${ }^{30}$ and so on. There have been made a progress in enhancing drug delivery with solid MNs for skin pretreatment. For example, Yan et al. studied the effect of MN height on the drug flux. ${ }^{31}$ And found that solid silicon MNs with needle height higher than $600 \mu \mathrm{m}$ generated higher acyclovir flux than those with needle height lower than $300 \mu \mathrm{m}$, and further increase of the needle height beyond the sufficient height $(600$ $\mu \mathrm{m})$ did not show a significant increase in drug flux. In addition, lower needle density were more effective in enhancing drug flux. Kalluri et al. utilized the DermaRoller ${ }^{\circledR}$ device with metal MNs to create microchannels in the skin. The microchannels could be closed within a few hours, depending on the height of MNs. ${ }^{32}$ Mohammed et al. studied the permeation of cosmeceutically relevant peptides in human skin pretreated by metal MNs. And concluded that the MN pretreatment enhancement could be effective when delivering some therapeutic peptides, but it is not a one size fits all solution for topical delivery. ${ }^{33}$

Although there are many studies utilizing polymer MNs for enhancing transdermal drug delivery, there are still some issues need to be addressed prior to its applications, such as systematic investigations on the mechanical and stability properties, various factors on the rate of drug permeation into the pretreated skin, the mass production of solid polymer MNs, and so on. In this work, solid polymer MN arrays with different dimensions and specifications were made out of biodegradable and biocompatibility polymer, the polylactic acid (PLA), which has been introduced to use as resorbable sutures in surgery, ${ }^{34}$ using a mold-based fabrication method. The mechanical and stability properties of the MNs were first investigated to figure out how many times of the MNs could be repeatly used, which is important for the future applications especially in cosmetics. Then the effect of different factors, including $\mathrm{MN}$ height and density, drug concentration, the viscosity of drug formulation, and the administration time of drug on the skin, on the drug permeation rate into the pretreated skin was systemically studied. The goal of this study is to figure out how we can optimize the above parameters to obtain an improved drug permeation rate into the skin with the pretreatment of solid polymer MNs. Finally, in order to better reflect the relationship of local drug absorption and microneedles, insulin was chosen as a model drug for transdermal absorption studies with different administration routes. All the results may provide the basis for future clinical and commercialization of solid polymer MNs for transdermal drug delivery.

\section{Materials and methods}

\subsection{Chemicals}

Polylactic acid was purchased from Lakeshore Biomaterials, Inc. (PLA, $1.0 \mathrm{dL} \mathrm{g}^{-1}$, Birmingham, AL). Sulforhodamine $\mathrm{B}$, insulin and sodium citrate buffer ( $\mathrm{PH}$ 4.5) were purchased from Sigma (St. Louis, MO, USA). Polydimethylsiloxane (PDMS, Sylgard 184), composed of Base Part A and Curing Agent Part B, was purchased from Dow Corning (Midland, MI). Phosphate buffer saline (PBS) tablets (PH 7.2-7.4) was purchased from M\&C Gene Technology Ltd. (Beijing, China). Polyvinyl alcohol (PVA, 75\% hydrolyzed, MW approximately 2000) was purchased from Acros Organics (New Jersey, USA). Streptozotocin was purchased by AbMole Bioscience (Shanghai, China). Porcine cadaver skins were purchased from a local slaughterhouse immediately after death.

\subsection{Fabrication of solid polymer microneedles}

To fabricate solid polymer MNs, a polydimethylsiloxane (PDMS, Sylgard 184, Dow Corning, Midland, MI) mold was initially fabricated using laser and molding techniques to produce a series of conical MN cavities with different dimensions. ${ }^{35}$ The PDMS mold was piled with pellets of biodegradable polylactic acid (PLA, $1.0 \mathrm{dL} \mathrm{g}^{-1}$, Birmingham Polymer, Pelham, AL) and placed in a vacuum oven under vacuum at $-85 \mathrm{kPa}$ and heated at $200^{\circ} \mathrm{C}$ for $2 \mathrm{~h}$ to make the polymer melt completely. The PLA MNs were then cooled to room temperature and removed from the mold for further use. The heights of the PLA MNs were 600, 700 and 800 $\mu \mathrm{m}$, and the densities were 100, 144, 196 and $256 \mathrm{MNs}$ per $\mathrm{cm}^{2}$.

\subsection{Mechanical stability of microneedles}

Multiple applications test were designed to assess the mechanical and stability properties of MNs and to determine their ability of multiple insertions in cosmetics. The porcine cadaver skin was used as the model for human skin for the insertion tests. Prior to the piercing experiments, porcine cadaver skin was processed by removing hair using a razor and subcutaneous fat using a scalpel with approval from the BUCT IACUC. Subsequently, the porcine skin samples were manually treated with $10 \times 10 \mathrm{MN}$ arrays with different heights $(600,700$ and $800 \mu \mathrm{m})$ and a fixed MN density of $100 \mathrm{MNs}$ per $\mathrm{cm}^{2}$ using a force of $5 \mathrm{~N}$ for 20 times. After removing the MNs from the skin, the model drug, sulforhodamine B, was applied onto the pretreated locations on the skin for $5 \mathrm{~min}$. The dye was then washed off using DI water. Finally, the porcine cadaver skins were imaged using a stereomicroscope (Olympus SZX7) with a digital camera (Olympus DP71). The percentage of successful penetration (the number of red spots divided by the total number of MNs per array) was finally calculated.

\subsection{In vitro and in vivo drug permeation studies}

To quantitatively evaluate the effects of $\mathrm{MN}$ dimensions on the drug permeation rate, the MNs with different heights of 600 , 
700 and $800 \mu \mathrm{m}$ and different densities of 100, 144, 196 and 256 MNs per $\mathrm{cm}^{2}$ were used to penetrate the porcine skins using a force of $5 \mathrm{~N}$. After the MN pretreatment, $8 \mu \mathrm{L}$ of sulforhodamine B with a concentration of $0.1 \mathrm{mg} \mathrm{mL}^{-1}$ was applied onto the skin and just covered the insertion sites and keep for $5 \mathrm{~min}$. The residual drug on the skin surface was then cleaned up using DI water for 3 times and collected for further measurement. The amount of drug permeated into the skin could be calculated by measuring the amount of drug collected above using a fluorescence microplate reader (Fluoroskan Ascent 374, Thermo Scientific).

In addition, the effects of drug concentration $(0.01,0.05,0.1$, $0.2,0.4,0.6,0.8$ and $1 \mathrm{mg} \mathrm{mL}{ }^{-1}$ ), the viscosity of drug solutions (controlled by mixing with $0 \%, 10 \%, 20 \%$ and $30 \% \mathrm{PVA}$ ), as well as the administration time of the drug (from $5 \mathrm{~min}$ to $6 \mathrm{~h}$ ) on the drug permeation rate were also studied using the same procedure mentioned above. While for the investigation of the effect of administration time of the drug on the drug permeation rate, the female BALB/c mice (6-8 weeks old, $16 \pm 0.7 \mathrm{~g}$ ) was used as administration model. The viscosities of drug solution were measured by NDJ-8S viscosity meter. The PLA MNs with a height of $600 \mu \mathrm{m}$ and a density of $100 \mathrm{MNs}$ per $\mathrm{cm}^{2}$ were used for this study. Throughout the whole experiment, the porcine skins were storied in phosphate buffered saline (PBS, PH 7.2-7.4, Macgene, Beijing, China) buffer to simulate the in vivo physiological environment.

\subsection{In vivo absorption studies}

To preferably indicate the relationship of local drug absorption and microneedles, insulin was selected as the specific drug model for in vivo transdermal delivery experiment. Prior to the experiment, the diabetic model was induced in BALB/c female mice (6-8 weeks old, $16 \pm 0.7 \mathrm{~g}$ ) with streptozotocin (200 mg $\mathrm{kg}^{-1}$ in sodium citrate buffer, $\mathrm{PH} 4.5$ ) administrated by intraperitoneal injection after they were fasted for $6 \mathrm{~h}$ only in case of water. Mice were counted as diabetics when their baseline glucose levels exceeded $16.7 \mathrm{mM}\left(300 \mathrm{mg} \mathrm{dL}^{-1}\right)$ after $48 \mathrm{~h}$. Blood glucose levels were measured with a glucose assay kit on a Blood Analyzer (ONETOUCH® UtraEasy ${ }^{\circledR}$, Shanghai, China).

The diabetic mice with blood glucose levels between 20 and $30 \mathrm{mM}$ were then selected for study and were divided in groups containing five mice each. The hairs on the skin surface of the abdomen and back of mice were shaved using electrical shearing knife and depilated with depilatory cream (Veet®, China) after they were anesthetized. Before application of the MNs, the blood glucose values of the mice were detected to be as the baseline glucose levels. Then the PLA MNs with a height of $600 \mu \mathrm{m}$ and a density of $100 \mathrm{MNs}$ per $\mathrm{cm}^{2}$ were used to penetrate the hairless back skins of the mice after disinfection. After the MN pretreatment, $100 \mu \mathrm{L}$ of insulin solution with a concentration of $3 \mathrm{IU} \mathrm{mL}{ }^{-1}$ was applied onto the skin and just covered the insertion sites for $1 \mathrm{~h}$. The insulin solution was then washed off using DI water. Blood glucose were withdrawn from the tail vein at $0,0.5,1,1.5,2,2.5,3,4,5,6,7$ and $8 \mathrm{~h}$ after the beginning of the experiments and blood glucose levels were measured. The drug transport studies were performed with different administration routes including transdermal insulin delivery using MNs
(3 IU mL ${ }^{-1}$ ), subcutaneous injection using hypodermic needle (20 IU $\mathrm{kg}^{-1}$ ) and transdermal insulin delivery with no MNs pretreatment $\left(3 \mathrm{IU} \mathrm{mL} \mathrm{m}^{-1}\right)$. Finally, in order to observe the changes in blood glucose levels with time, a time controlled group was added. All animals were maintained in accordance with the guidelines of the Zoological Institute of China, and approved by the institutional ethical committee (IEC) of Beijing University of Chemical Technology.

\subsection{Statistical analysis}

All results were expressed as mean \pm standard error of mean. Statistical analysis was performed using analysis of variance followed by Tukey-Kramer multiple comparisons test or unpaired two-tailed student- $t$-test using GraphPad Instat software. $P<0.05$ was considered to be statistically significant.

\section{Result and discussion}

\subsection{Fabrication of solid polymer microneedles}

As a starting point, solid polymer MN arrays were fabricated with PLA using thermal micromolding technique, as shown in Fig. 1. Each MN array occupied a square of $1 \mathrm{~cm}^{2}$ area containing MNs with different number, density and height. The dimensions of MNs were designed according to the needs of particular study. Three different MN arrays with the same density of $100 \mathrm{MNs}$ per $\mathrm{cm}^{2}$ and different heights of 600 (Fig. 1A and D), 700 (Fig. 1B and E) and $800 \mu \mathrm{m}$ (Fig. 1C and E) were prepared to investigate the effect of $\mathrm{MN}$ height on the mechanical stability and the drug permeation rate. To further investigate the effect of $\mathrm{MN}$ dimensions on drug permeation into the skin, MN arrays with the same height of $600 \mu \mathrm{m}$ and different densities of 144, 196 and $256 \mathrm{MNs}$ per $\mathrm{cm}^{2}$ were also prepared, as shown in Fig. 1G-I. In addition, the MN array with a height of $600 \mu \mathrm{m}$ and a density of $100 \mathrm{MNs}$ per $\mathrm{cm}^{2}$ (Fig. 1A) was used to evaluate the effect of drug concentration, the viscosity of drug formulation and the administration time on the drug permeation rate into the skin.

\subsection{Mechanical stability of microneedles}

In order to assess the mechanical stability of MNs (i.e. the ability of multiple insertions into the skin), which is important for the future applications in cosmetics, multiple insertions test were performed with MNs with different heights of 600, 700 and 800 $\mu \mathrm{m}$. The mechanical stability of the MNs could be evaluated by the relationship between the percentage of successful insertions and the number of MNs on a patch. As shown in Fig. 2, the red dots remained on the surface of the skin indicated the drug diffusion into the skin through the micropores created by the MNs, which means the successful insertion of the MN. By counting the red dots under a microscope, the percentage of successful insertions per array was calculated, as shown in Fig. 2.

For the first 3 insertions, all the three kinds of MNs achieved almost $100 \%$ successful insertions, indicating the initial good mechanical property of the MNs. Further increase in the number of $\mathrm{MN}$ applications on the porcine cadaver skin resulted in a decrease in the percentage of successful insertions for 

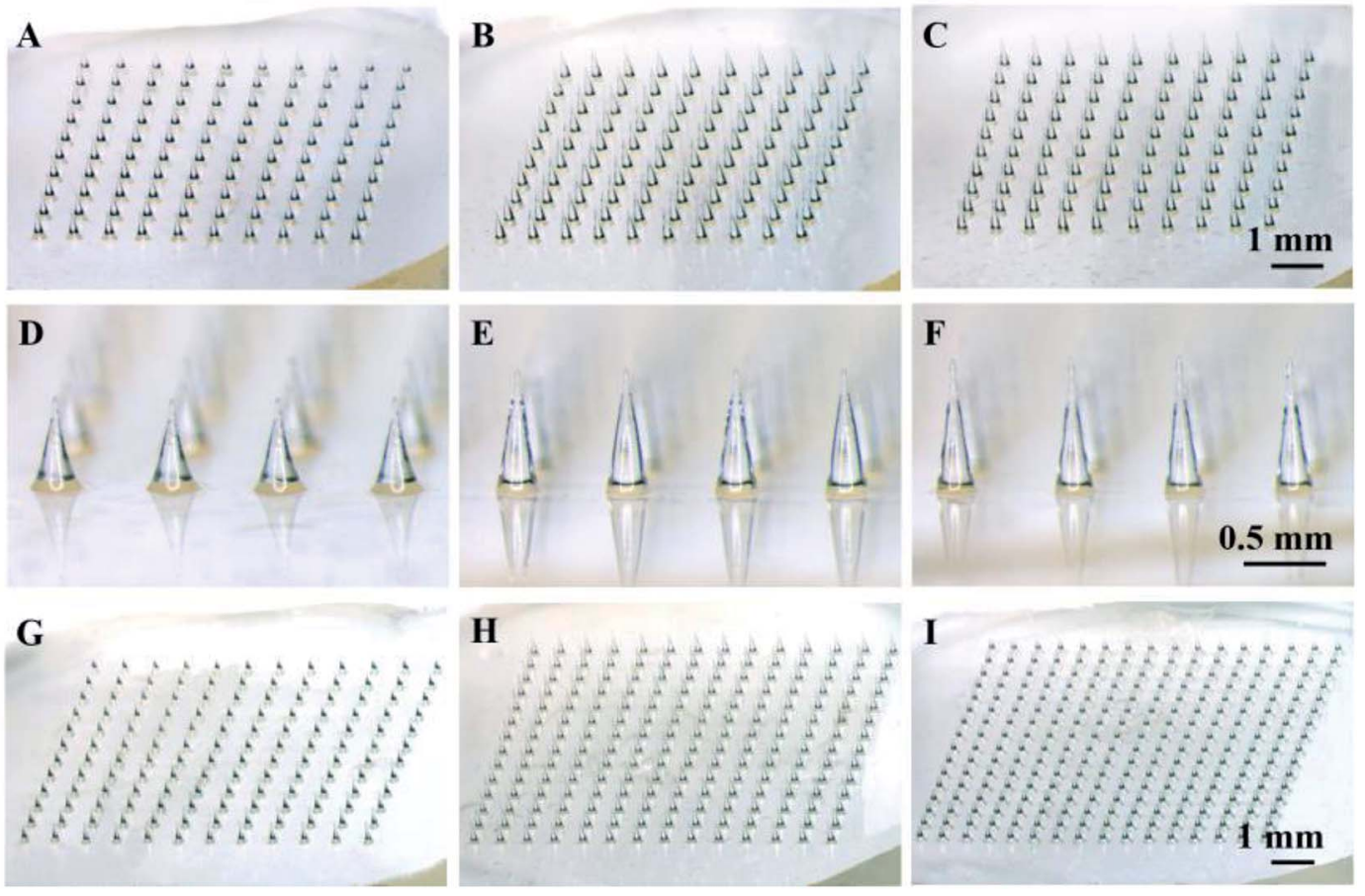

Fig. 1 Light micrograph of PLA MNs, as viewed from both (A-C, G-I) above and (D-F) side. (A, D) $600 \mu \mathrm{m} \times 100 \mathrm{MNs}$ per $\mathrm{cm}^{2},(B, E) 700 \mu \mathrm{m} \times$ $196 \mathrm{MNs}$ per $\mathrm{cm}^{2}$, (C, F) $800 \mu \mathrm{m} \times 100 \mathrm{MNs}$ per $\mathrm{cm}^{2}$, (G) $600 \mu \mathrm{m} \times 144 \mathrm{MNs}$ per cm², (H) $600 \mu \mathrm{m} \times 196 \mathrm{MNs}$ per $\mathrm{cm}^{2}$, (I) $600 \mu \mathrm{m} \times 256 \mathrm{MNs}$ per $\mathrm{cm}^{2}$, where values indicate $M N$ height $\times$ array density.

all MNs. For the MNs with a height of 700 and $800 \mu \mathrm{m}$, the percentage of successful insertions decreased to be $78 \%$ and $70 \%$ respectively at the $10^{\text {th }}$ insertion. While for the MNs with a height of $600 \mu \mathrm{m}$, the percentage of successful insertions was approximately $93 \%$, which is higher than those induced by longer MNs. With further increase of the number of insertions, the percentage of successful insertions promptly decreased to be lower than $20 \%$ for the MNs with a height of 700 and $800 \mu \mathrm{m}$.

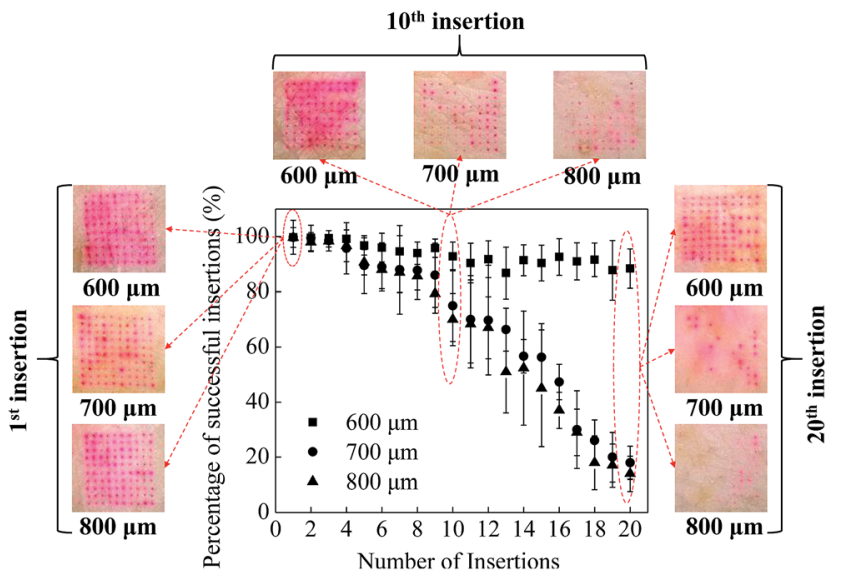

Fig. 2 Relationship between percentage of successful insertions and the number of insertions with MNs with different heights. The microscopic images of porcine skin treated with the MNs at $1^{\text {st }}, 10^{\text {th }}$ and $20^{\text {th }}$ insertion are provided on the left, top and right of the graph respectively. Each data point represents the average of 5 experiments. Standard deviation bars are shown.
However, the MNs with a height of $600 \mu \mathrm{m}$ still achieved $90 \%$ successful insertions. Although the longer MNs (i.e. 700 and 800 $\mu \mathrm{m}$ in this work) have sharp MN tips and good mechanical property, with multiple insertions, some MN tips are easy to be bent due to the long body of the MNs. The bent MNs are hard to penetrate the skin which could decrease the percentage of successful insertions. For short MNs (i.e. $600 \mu \mathrm{m}$ in this work), the MN tips are not easy to be bent due to the short body of the MNs, leading to a good mechanical stability of the short MNs.

\subsection{Effect of microneedle dimensions on drug permeation into the skin}

As known that MN height and density play an important role in enhancing drug permeation into the skin. To investigate the effect of MN dimensions on the amount of drug permeation, porcine cadaver skins were treated with MN array with different heights ( 0 (control, no MNs on the patch), 600, 700 and $800 \mu \mathrm{m}$ ) and densities (100, 144, 196 and $256 \mathrm{MNs}$ per $\left.\mathrm{cm}^{2}\right)$. As shown in Fig. 3A, the skin pretreatment with MNs could significantly enhance the drug permeation into the skin by 11-17 folds as compared to the control group (i.e. no MNs on the patch). For the control group, the amount of drug permeation into the skin was only $14 \mathrm{ng} \mathrm{cm}{ }^{-2}$ in $5 \mathrm{~min}$ from $0.1 \mathrm{mg} \mathrm{mL}^{-1}$ sulforhodamine B solution. While for the pretreatment with $600 \mu \mathrm{m} \mathrm{MNs,}$ the drug permeation amount was $152 \mathrm{ng} \mathrm{cm}{ }^{-2}$. With further increase in the MN height to be 700 and $800 \mu \mathrm{m}$, the drug permeation amount increased slightly to be 208 and $238 \mathrm{ng}$ $\mathrm{cm}^{-2}$. This indicated that MNs can penetrate across the stratum corneum and create pathways for enhancing drug transport. 

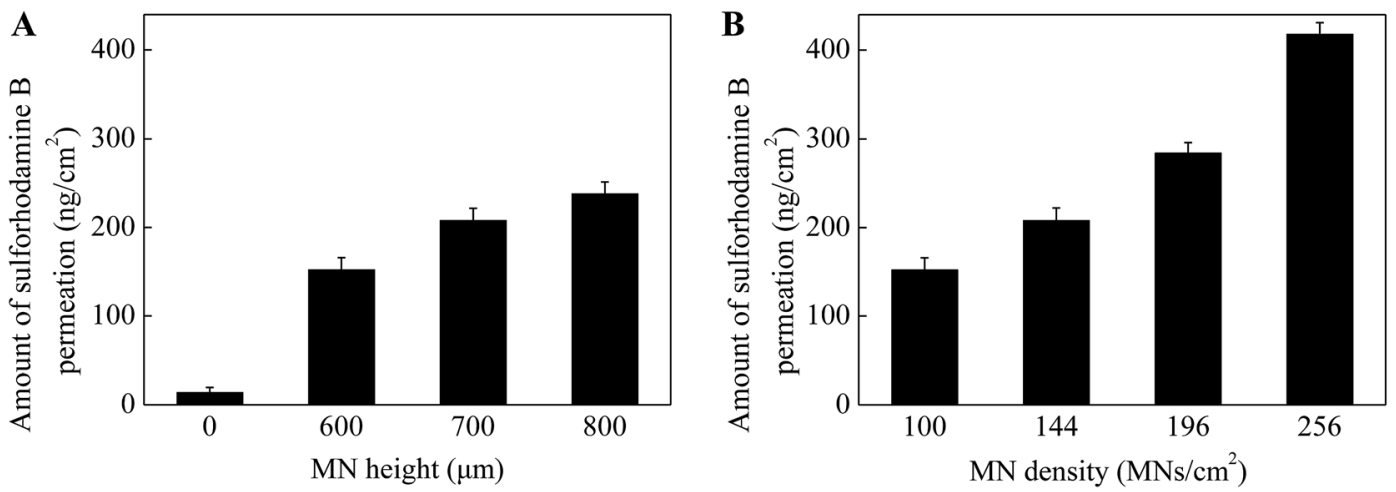

Fig. 3 The amount of sulforhodamine B permeated into the porcine skin treated with MNs with (A) different heights (600, 700 and $800 \mu \mathrm{m})$ and (B) densities $\left(100,144,196\right.$ and $256 \mathrm{MNs}$ per $\left.\mathrm{cm}^{2}\right)$ in $5 \mathrm{~min}$ from $0.1 \mathrm{mg} \mathrm{mL}^{-1}$ surforhodamine B solution. Each data point represents the average of 5 experiments. Standard deviation bars are shown.

Also longer MNs could induce deeper and bigger micro-holes in the skin and eventually more drug could permeate into the skin.

In addition to the MN heights, the effect of MN densities is also important in enhancing drug permeation into the skin. As shown in Fig. 3B, the drug permeation amount was $152 \mathrm{ng} \mathrm{cm}^{-2}$ after pretreated with the MNs with the density of 100 MNs per $\mathrm{cm}^{2}$. With the increase of MN densities from 100 to 144, 196 and $256 \mathrm{MNs}$ per $\mathrm{cm}^{2}$, the drug permeation amount increased to be 210,284 and $418 \mathrm{ng} \mathrm{cm}^{-2}$ respectively. As discussed above, the micro-holes created by the microneedles are the main reason to enhance the drug permeation into the skin. The MN patches with higher MN densities had more MNs on the patches with the same size. That is, higher densities of the MNs, more microholes could be created by the MNs, resulting in more drug permeated though the micro-holes into the skin. In addition, to better understand the effect of MN densities on drug permeation, the local magnification images of PLA MNs with different densities and the skins after pretreating with corresponding MNs were shown in Fig. 4. The red dots on the images of the skins demonstrated that the micro-holes created by the MNs could effectively enhance the drug permeation into the skin. Thus, the MN patches with higher MN densities could induce higher drug permeation into the skin.

Based on the results above, only in consideration of increasing drug permeation amount, the MNs with $800 \mu \mathrm{m}$ in height and 256 MNs per $\mathrm{cm}^{2}$ are more appropriate for the skin pretreatment as compared to the MNs with shorter heights and lower densities. But an ideal MN patch could also not cause any pain or bleeding. According to previous reports, ${ }^{36} \mathrm{MN}$ length had the strongest effect on pain, where a 3-fold increase in length increased the pain score by 7 -fold. And a 10 -fold increase in the density of MNs increased pain over 2-fold. Therefore, an optimized MN patch in future applications should consider the balance between the effectiveness and the pain associated with insertions.

\subsection{Effect of drug concentration on drug permeation into the skin}

Drug permeation amount and rate are two important factors in the application of MNs. In addition to the MN dimensions, another method to control the drug permeation is drug concentration. To investigate the effect of drug concentration on the drug permeation, a series of drug solutions with the concentrations of $0.01,0.05,0.1,0.2,0.4,0.6,0.8$ and $1 \mathrm{mg} \mathrm{mL}^{-1}$ were applied on the pretreated porcine cadaver skin for $5 \mathrm{~min}$. The MNs used for the skin pretreatment in this work had a height of $600 \mu \mathrm{m}$ and a density of $100 \mathrm{MNs}$ per $\mathrm{cm}^{2}$. As shown in Fig. 5, the amount of drug permeation (square) increased linearly with an increase of drug concentration from 0.01 to $1 \mathrm{mg} \mathrm{mL}{ }^{-1}$. For the administration of $0.01 \mathrm{mg} \mathrm{mL}^{-1} \mathrm{drug}$, the amount of drug permeation was $15 \mathrm{ng} \mathrm{cm}^{-2}$ in $5 \mathrm{~min}$. While as the drug concentration increased to be $1 \mathrm{mg} \mathrm{mL}^{-1}$ (i.e. 100 fold), the drug permeation amount increased to be $1568 \mathrm{ng}$ $\mathrm{cm}^{-2}$ (i.e. also about 100-fold). However, the drug permeation rate (triangle) was almost kept at a steady level of $20 \%$ following with the variations of drug concentration from 0.01 to $1 \mathrm{mg}$ $\mathrm{mL}^{-1}$. As known that the sulforhodamine $\mathrm{B}$ is a small molecule drug with the molecule weight of $580 \mathrm{Da}$. With the increase of drug concentration, the amount of drug applied on the pretreated skin increased and then the amount of drug permeated into skin through the microconduits created by MNs within the same time would also increase accordingly. While for the small molecule drug, the change of drug concentration has little influence on the viscosity of drug solution. Thus, the drug flow rate would not be affected and then the rate of drug permeated into the skin remained relatively unchanged. Therefore, the amount of drug permeation in a specific time could be well controlled by changing the drug concentrations without altering the drug permeation rate.

\subsection{Effect of the viscosity of drug solution on drug permeation into the skin}

As mentioned above, the drug solution flow rate was influenced by the viscosity of drug solution and then the permeation of drug into the skin was also affected. Hence, the viscosity of drug solution is also an important factor in enhancing drug permeation into the skin. To investigate the effect of the viscosity of drug solution on the drug permeation, a series of drug solutions with the viscosities of $1.2,30,600$ and $3700 \mathrm{mPa} s$ were 

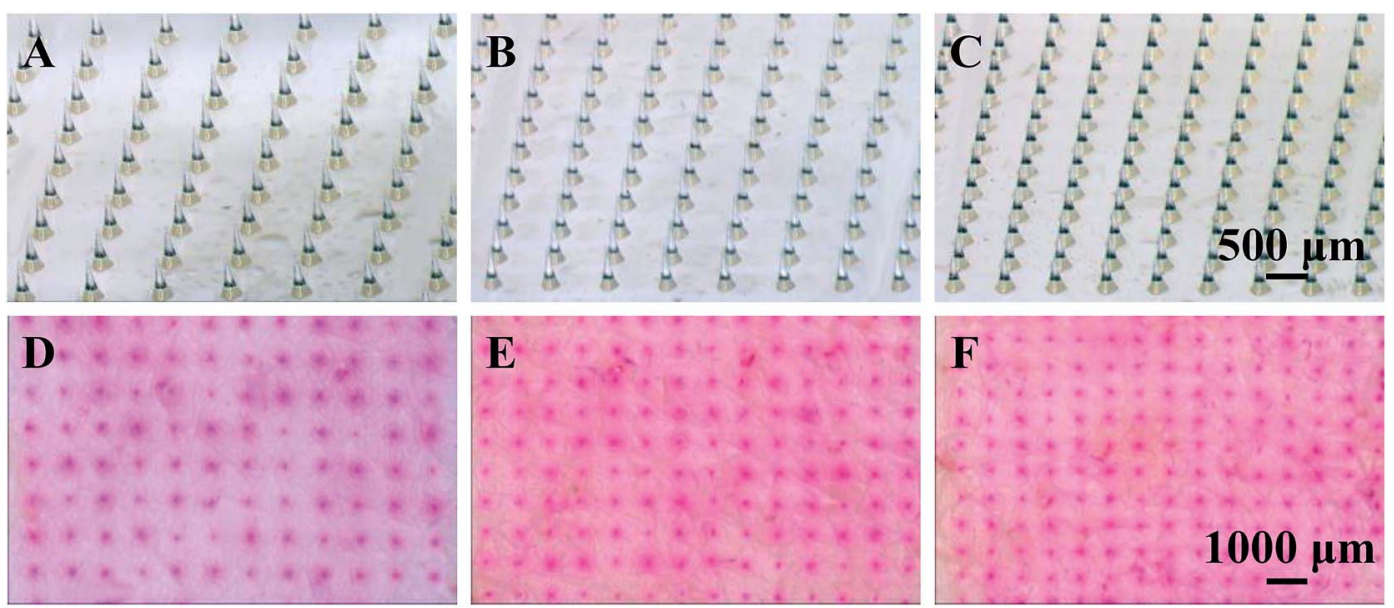

Fig. 4 Microscopic images showing the enlarging structure of the fabricated PLA MNs and the piercing effect of the MNs. (A-C) MN arrays with a height of $600 \mu \mathrm{m}$ and different densities of (A) $144 \mathrm{MNs}$ per $\mathrm{cm}^{2}$, (B) $196 \mathrm{MNs}$ per $\mathrm{cm}^{2}$ and (C) $256 \mathrm{MNs}$ per $\mathrm{cm}^{2}$. (D-F) The porcine skin samples treated with MNs with different densities of (D) $144 \mathrm{MNs}$ per $\mathrm{cm}^{2}$, (E) $196 \mathrm{MNs}$ per $\mathrm{cm}^{2}$ and (F) $256 \mathrm{MNs}$ per $\mathrm{cm}^{2}$.

prepared, which was controlled by mixing with $0 \%, 10 \%, 20 \%$ and 30\% PVA, respectively (Fig. 6 triangle). And then they were applied onto the penetrated skin and kept for $5 \mathrm{~min}$. The MNs with a height of $600 \mu \mathrm{m}$ and a density of $100 \mathrm{MNs}$ per $\mathrm{cm}^{2}$ were used for the skin pretreatment in this study. As shown in Fig. 6, for the drug solution without PVA (i.e. the concentration of PVA was 0 ), the viscosity of drug solution was $1.2 \mathrm{mPa}$ s and the drug permeation amount was $152 \mathrm{ng} \mathrm{cm}^{-2}$ in $5 \mathrm{~min}$. When the PVA concentration increased to $10 \%$, the viscosity of drug solution slightly increased to $30 \mathrm{mPa}$ s and the drug permeation amount

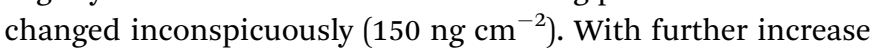
of PVA concentration to $20 \%$ and $30 \%$, the viscosity of drug solution increased to 600 and $3700 \mathrm{mPa}$ s. Accordingly, the amount of drug permeation decreased to 125 and $108 \mathrm{ng} \mathrm{cm}^{-2}$, respectively. This was due to the fact that with the increase of the viscosity of drug solution, the drug solution becomes increasingly thick and then the fluidity of drug solution gradually declines. Thus, the flow resistance of the drug solution

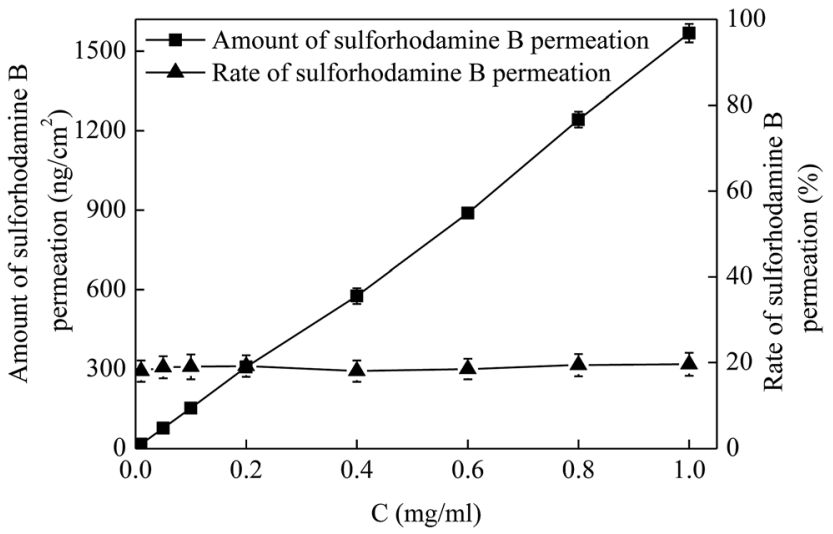

Fig. 5 The permeation profiles of sulforhodamine B with different concentrations across porcine skin samples treated with MNs. Each data point represents the average of 5 experiments. Standard deviation bars are shown. increased accordingly in the process of the drug permeation into skin, leading to a lower rate of the drug solution flow through the microchannels in the skin. And this eventually resulted in an obvious decrease of the amount of drug permeation into skin in a specific time. Therefore, the amount of drug permeation could be controlled effectively by adjusting the viscosity of drug solution according to the demand of the required dosage.

\subsection{Effect of administration time on drug permeation into the skin in vivo}

The administration time of the drug solution on the skin surface is also an important factor that affect the drug permeation. In this work, $0.1 \mathrm{mg} \mathrm{mL}^{-1}$ sulforhodamine B solution was applied on the pretreated mice skin in vivo (Fig. 7A) and kept for $5 \mathrm{~min}, 10 \mathrm{~min}, 30 \mathrm{~min}, 1 \mathrm{~h}, 2 \mathrm{~h}, 4 \mathrm{~h}$ and $6 \mathrm{~h}$. MN arrays with

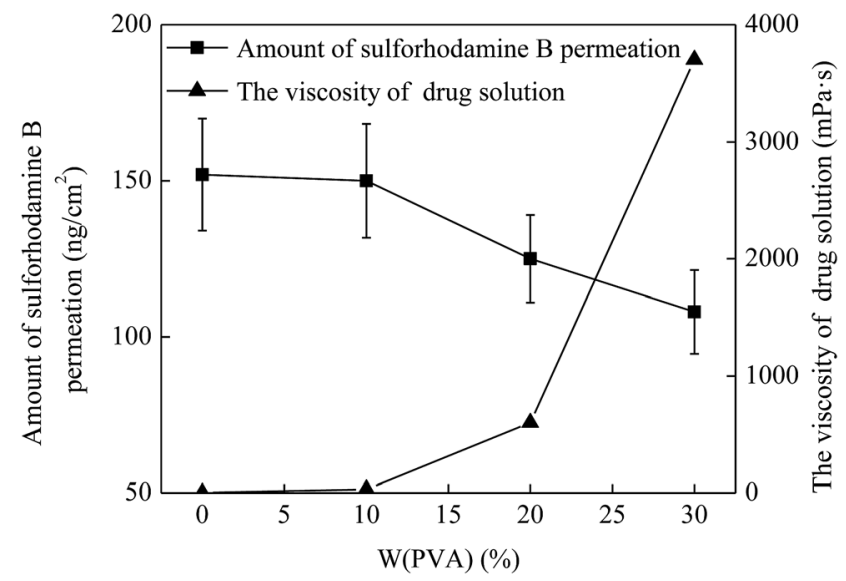

Fig. 6 The amount of sulforhodamine B permeated into the skin (square) and the viscosities of drug solutions (triangle) with different PVA concentrations. Each data point represents the average of 5 experiments. Standard deviation bars are shown. 
a height of $600 \mu \mathrm{m}$ and a density of $100 \mathrm{MNs}$ per $\mathrm{cm}^{2}$ were used in this test. As shown in Fig. 7B, with the increase of the administration time of the drug solution, the amount of drug permeated into the mouse skin increased accordingly. In the initial $1 \mathrm{~h}$, the drug permeation amount increased rapidly from 0 to be about $530 \mathrm{ng} \mathrm{cm}^{-2}$. While after $1 \mathrm{~h}$, the drug permeation amount began to level off. One explanation for the observed results were due to the recovery of the pore created by MNs on the skin as the administration time of drug prolonged. And our previous study has been verified that the micro-holes created by the MNs would recover completely at about $1 \mathrm{~h}$ after MN pretreatment. ${ }^{37}$ Once the micro-holes on the mouse skin created by MNs healed, drug molecules would be difficult to diffuse rapidly into the skin across the micro-holes. In addition, to better illustrate the effect of administration time on drug permeation into skin in vivo, the microscopic images of mice skin treated with MNs after the drug administration time of $5 \mathrm{~min}, 10 \mathrm{~min}, 30 \mathrm{~min}, 1 \mathrm{~h}, 2 \mathrm{~h}, 4 \mathrm{~h}$ and $6 \mathrm{~h}$ are provide on the graph. The color changes of red dots on the images of the skins reflected the changes of drug concentration in accordance with the above results. Thus, the amount of drug permeated into the skin no longer substantially increased and mainly remained unchanged after $1 \mathrm{~h}$. Therefore, the drug permeation amount could increase effectively mainly in the initial $1 \mathrm{~h}$ after the skin pretreated with the MNs.

\subsection{In vivo absorption studies}

In order to more intuitively reflect the effect of MNs pretreatment on enhancing drug absorption in vivo, three administration routes including transdermal insulin delivery using MNs (3 IU $\mathrm{mL}^{-1}$ ), subcutaneous injection using hypodermic needle (20 IU $\mathrm{kg}^{-1}$ ) and transdermal insulin delivery with no MNs pretreatment ( $3 \mathrm{IU} \mathrm{mL}^{-1}$ ) were designed in this work. The diabetic mice without any treatment were treated as a control group. Blood glucose were then monitored at $0,0.5,1,1.5,2,2.5,3,4,5,6,7$ and $8 \mathrm{~h}$ after the beginning of the experiments. As shown in Fig. 8, for the time-controlled group, the blood glucose level was almost kept a steady level $100 \%$ with a small fluctuation. And

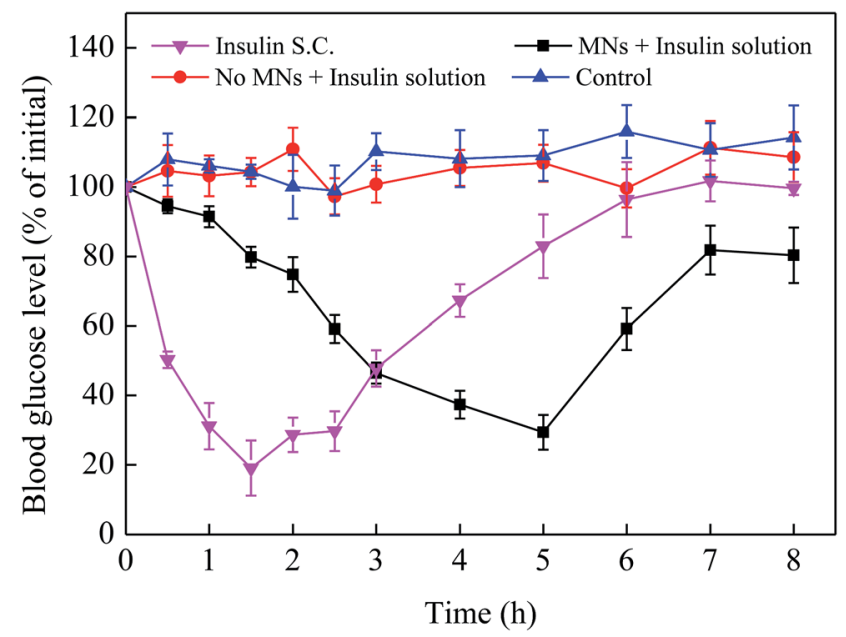

Fig. 8 Blood glucose levels as a percentage of the initial value in mice after subcutaneous hypodermic insertions of insulin $(\boldsymbol{\nabla})$, transdermal insulin delivery using MNs ( $\mathbf{0})$, transdermal insulin delivery without MNs pretreatment $(\mathbf{O})$ and time control $(\boldsymbol{\Delta})$. Each data point represents the average of 5 experiments. Standard deviation bars are shown.

compared with the control group, there was no significant difference in the variations of the blood glucose level of the group where insulin solution was applied onto the surface of mice skin. While for the subcutaneous injection of insulin, the blood glucose level decreased rapidly from $100 \%$ to $19 \%$ in the initial $1.5 \mathrm{~h}$ and then gradually increased to about $100 \%$ after $1.5 \mathrm{~h}$. For the MNs group, the minimum blood glucose level was about $29 \%$ of its initial value at $5 \mathrm{~h}$, which is remarkably different from the group without MNs pretreatment. This was due to the fact that the MNs can produce microchannels for drug delivery and then enhance the drug active ingredient penetration into skin compared with transdermal route. While after the MNs pretreatment, the drug penetrated slowly into the skin rather than quickly and directly into the body like the hypodermic needles injection. Thus, the subcutaneous injection of insulin can reduce the blood glucose quickly while the microneedle-
A

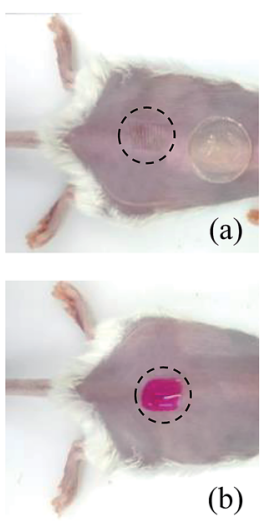

B

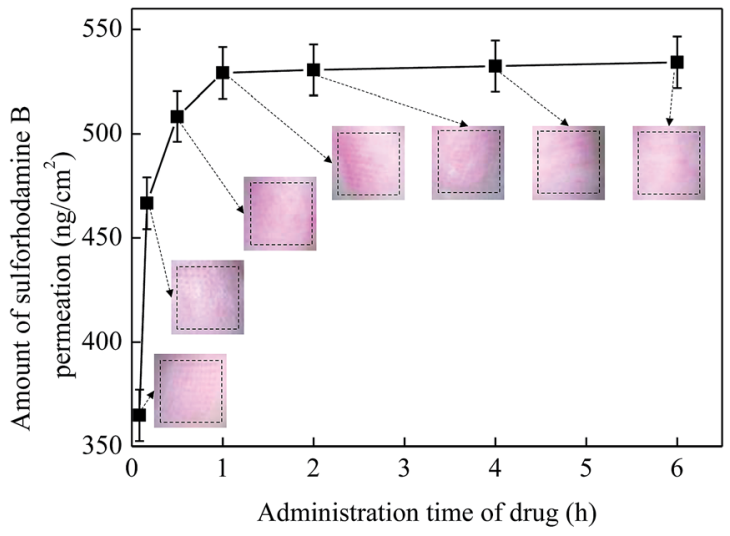

Fig. 7 (A) The images of a mouse skin in vivo pretreated using a solid PLA microneedle patch before (a) and after (b) applying sulforhodamine B solution. (B) The permeation profiles of sulforhodamine B with different dwell time across mice skin pretreated with MNs. Each data point represents the average of 5 experiments. Standard deviation bars are shown. 
mediated insulin delivery is slow to achieve the effect of lowering the blood glucose. Therefore, the $\mathrm{MN}$ is an effective way to promote transdermal drug absorption.

\section{Conclusions}

In conclusion, this study demonstrated that different dimensions of polymer microneedles were fabricated to enhance transdermal drug delivery aimed at small molecule drugs in cosmetics. We observed that the excellent mechanical stability of MNs with a height of $600 \mu \mathrm{m}$ made it more viability in the skin pretreatment than MNs with a height of 700 and $800 \mu \mathrm{m}$. In addition, MN arrays with higher height and density resulted in greater drug permeability compared with no MNs pretreatment. We also observed that there was a linear relationship between the amount of drug permeation and drug concentration owing to the unchanged drug viscosity. However, the increasing of drug viscosity could reduce the amount of drug permeation. It was shown that the microchannels created by MNs were viable for enhancing drug delivery within $1 \mathrm{~h}$ by pharmacokinetic evaluation. The enhanced transdermal absorption of insulin was also demonstrated in vivo by effective control of blood glucose level. Altogether, these consequences show that optimization the dimensions of biodegradable and biocompatible polymer MNs can facilitate drug permeation. The sufficient combination of MNs pretreatment and the effect of small molecule drugs on drug permeation is also practically significant for either clinical application or cosmetic industry.

\section{Acknowledgements}

This work was financially supported by the National Natural Science Foundation of China (51473017, 51673019), the Fundamental Research Funds for the Central Universities (buctrc201406), the Innovation and Promotion Project of Beijing University of Chemical Technology, and the long-term subsidy mechanism from the Ministry of Finance and the Ministry of Education of PRC.

\section{References}

1 M. R. Prausnitz and R. Langer, Nat. Biotechnol., 2008, 26, 1261. 2 M. R. Prausnitz, S. Mitragotri and R. Langer, Nat. Rev. Drug Discovery, 2004, 3, 115.

3 K. Ita, Pharmaceutics, 2015, 7, 90.

4 Y. B. Choy and M. R. Prausnitz, Pharm. Res., 2011, 28, 943. 5 K. B. Ita, Curr. Drug Delivery, 2015, 12, 645.

6 J. Sawyer, S. Febbraro, S. Masud, M. A. Ashburn and J. C. Campbell, Br. J. Anaesth., 2009, 102, 210.

7 T. W. Wong, J. Controlled Release, 2014, 193, 257.

8 P. Charoenputtakun, S. K. Li and T. Ngawhirunpat, Int. J. Pharm., 2015, 495, 318.

9 B. Zorec, J. Jelenc, D. Miklavcic and N. Pavselj, Int. J. Pharm., 2015, 490, 65.

10 D. Park, H. Park, J. Seo and S. Lee, Ultrasonics, 2014, 54, 56.

11 C. Gomez, M. Benito, J. M. Teijon and M. D. Blanco, Ther. Delivery, 2012, 3, 373.
12 H. Marwah, T. Garg, A. K. Goyal and G. Rath, Drug Delivery, 2016, 23, 564.

13 Y.-C. Kim, J.-H. Park and M. R. Prausnitz, Adv. Drug Delivery Rev., 2012, 64, 1547.

14 S. Henry, D. V. McAllister, M. G. Allen and M. R. Prausnitz, J. Pharm. Sci., 1998, 87, 922.

15 X. X. Yan, J. Q. Liu, S. D. Jiang, B. Yang and C. S. Yang, Microelectron. Eng., 2013, 111, 33.

16 M. C. Gower, Opt. Express, 2000, 7, 56.

17 T. Omatsu, K. Chujo, K. Miyamoto, M. Okida, K. Nakamura, N. Aoki and R. Morita, Opt. Express, 2010, 18, 17967.

18 A. A. Fomani and R. R. Mansour, Sens. Actuators, A, 2011, 168, 233.

19 S. D. Gittard, A. Ovsianikov, N. A. Monteiro-Riviere, J. Lusk, P. Morel, P. Minghetti, C. Lenardi, B. N. Chichkov and R. J. Narayan, J. Diabetes Sci. Technol., 2009, 3, 304.

20 R. F. Donnelly, R. Majithiya, T. R. R. Singh, D. I. J. Morrow, M. J. Garland, Y. K. Demir, K. Migalska, E. Ryan, D. Gillen, C. J. Scott and A. D. Woolfson, Pharm. Res., 2011, 28, 41.

21 J. W. Lee, M. R. Han and J. H. Park, J. Drug Targeting, 2013, 21, 211.

22 D. V. McAllister, P. M. Wang, S. P. Davis, J. H. Park, P. J. Canatella, M. G. Allen and M. R. Prausnitz, Proc. Natl. Acad. Sci. U. S. A., 2003, 100, 13755.

23 H. R. Li, Y. S. J. Low, H. P. Chong, M. T. Zin, C. Y. Lee, B. Li, M. Leolukman and L. F. Kang, Pharm. Res., 2015, 32, 2678.

24 W. Martanto, S. P. Davis, N. R. Holiday, J. Wang, H. S. Gill and M. R. Prausnitz, Pharm. Res., 2004, 21, 947.

25 S. Xie, Z. J. Li and Z. Q. Yu, J. Drug Delivery Sci. Technol., 2015, 28, 11.

26 S. Liu, M. N. Jin, Y. S. Quan, F. Kamiyama, H. Katsumi, T. Sakane and A. Yamamoto, J. Controlled Release, 2012, 161, 933.

27 W. Luangveera, S. Jiruedee, W. Mama, M. Chiaranairungroj, A. Pimpin, T. Palaga and W. Srituravanich, J. Mech. Behav. Biomed. Mater., 2015, 50, 77.

28 M. C. Chen, M. H. Ling and S. J. Kusuma, Acta Biomater., 2015, 24, 106.

29 J. H. Oh, H. H. Park, K. Y. Do, M. Han, D. H. Hyun, C. G. Kim, C. H. Kim, S. S. Lee, S. J. Hwang, S. C. Shin and C. W. Cho, Eur. J. Pharm. Biopharm., 2008, 69, 1040.

30 Z. L. Xiang, H. Wang, A. Pant, G. Pastorin and C. Lee, Biomicrofluidics, 2013, 7, 10.

31 G. Yan, K. S. Warner, J. Zhang, S. Sharma and B. K. Gale, Int. J. Pharm., 2010, 391, 7.

32 H. Kalluri, C. S. Kolli and A. K. Banga, AAPS J., 2011, 13, 473. 33 Y. H. Mohammed, M. Yamada, L. L. Lin, J. E. Grice, M. S. Roberts, A. P. Raphael, H. A. E. Benson and T. W. Prow, PLoS One, 2014, 9, 9.

34 C. G. Ambrose and T. O. Clanton, Ann. Biomed. Eng., 2004, 32, 171.

35 Q. L. Wang, D. D. Zhu, Y. Chen and X. D. Guo, Mater. Sci. Eng. C, 2016, 65, 135.

36 H. S. Gill, D. D. Denson, B. A. Burris and M. R. Prausnitz, Clin. J. Pain, 2008, 24, 585.

37 D. D. Zhu, Q. L. Wang, X. B. Liu and X. D. Guo, Acta Biomater., 2016, 41, 312. 